Faculty of Philosophy

\title{
ANIMAL SOUNDS AS REPORTING VERBS IN TRANSLATIONS FROM ENGLISH INTO BCMS **
}

This paper discusses the use of animal-sound reporting verbs (e.g. grunt, bark, croak) in English literary texts and the significance they are assigned in Serbian translations. Based on a corpus of Dickens's novels Oliver Twist, David Copperfield, and Great Expectations, an analysis of animal-sound reporting verbs will be carried out in the SL and the TL texts for the purpose of learning whether the animal trait is (accurately) preserved in translations. Firstly, the translations of these verbs will be subject to componential analysis to determine if they retained the animal feature. The next step is checking if the translation solutions that do retain the animal feature accurately reflect the sound. Finally, translations that fail to convey the animal feature and the intended sound will be analyzed in order to determine the translation procedure employed. The preliminary hypothesis is that animal-sound verbs will largely be translated as their equivalent verbs (e.g. growls=reži). This research is important because animal-sound reporting verbs often serve as stylistic devices and offer glimpses into fictional characters' nature. Additionally, since few studies exist on the translation of reporting verbs from English into BCMS, this paper could draw more focus on them and shed more light on this specific matter.

Keywords: noise verbs, reporting verbs, characterization, translation procedures, animal sounds

\section{INTRODUCTION}

Reporting verbs (hereafter RVs) with visceral elements can aid in the textual representation of a literary character by lending nuance to their personation, indicating various aspects of spoken language that cannot be immediately discerned by, for example, neutral RVs such as "say" or "tell". Taking into consideration the

\footnotetext{
*emapandrc@gmail.com

** Presented at ELALT 5, March 2019.
} 
restrictive characteristics of text when it comes to conveying such elements of spoken communication (Brüngel-Dittrich, 2005), questions arise regarding the approach to reporting verbs reflecting animal sounds (e.g. hiss or chirp, hereafter ASRVs) in the process of translation. Put more precisely, the focus of this paper lies in whether such limitations of the written medium imply the vulnerability of the animal feature of the aforementioned RVs and whether this particular feature is more prone to be disregarded in translation from English into Serbian.

The initial hypothesis is that, due to their significance and contribution to the emotional coloring and the overall image and perception of literary characters, ASRVs are far more likely to be translated into Serbian with their exact equivalents rather than with non-animal RVs. Therefore, a given animal feature is retained in the target language as well and the intended characterization preserved. The matter at hand can also be viewed in the following manner: this paper juxtaposes the restrictiveness of the written medium with respect to spoken-language aspects and the translator's aim to remain as faithful to the original as possible and attempts to discover if the former affects the latter.

\section{THEORY, IMPLICATIONS AND QUESTIONS}

In the context of the written medium, the misinterpretation of messages, as stated by Silk, does happen despite an author's efforts to achieve maximum clarity. He goes on to link such miscommunications to the written medium's inability to "convey the mood, tone, emphasis, or body language of the author" (2007: 90).

When it comes to researching the theoretical background of ASRVs, perhaps one of the most crucial steps would be to see how they are treated in different existing taxonomies due to their paralinguistic feature. Among such classifications is one elaborated by Caldas-Coulthard, focusing on five separate kinds of RVs: descriptive, illocutionary, neutral, structuring, and discourse signaling. ASRVs would be placed in the descriptive category-what sets them apart from the other four RV types is the fact that they affect the manner in which a message is being conveyed (for example, sound-wise), rather than its content (Caldas-Coulthard, 1987: 162).

This contrast of content and manner of speaking is also present in the division of speech-act verbs which was established by Leech. In his work, the author differentiates between neutral, phonically descriptive, and contentdescriptive verbs (1983: 214). Not surprisingly, animal sound RVs such as growl, bark, or roar, fall under the category of phonically descriptive verbs, which the 
author also refers to as 'noise' verbs, due to their description of the manner of speaking (1983: 214).

Noise verbs carry paralinguistic speech-features, which, according to Brown, are a given speaker's means of indicating their attitude to interlocutors more clearly. Therefore, if we take into account that "paralinguistic vocal features will reinforce what the speaker says" (1990: 113), and their unquestionable effect on and involvement in what Brown refers to as the affective meaning (as opposed to the conceptual), the significance of 'noise' verbs in the development of a literary character's image becomes even more noticeable. ASRVs are no exception here.

The literary contribution of animal sounds and the RVs associated with them is easily recognized in the works of the Victorian author Charles Dickens. Namely, Dickens was keen on using phonically descriptive verbs as a way of emphasizing certain portrayals of his two-dimensional characters. Because of this tendency, linguists have studied the varying patterns of the employment of noise RVs in his novels.

As stated by Pablo Ruano San Segundo (2018), Dickens's utilization of such RVs was far from random, as the author frequently relied on attributing certain personality traits to his characters through their speech by coloring it with carefully chosen 'noise' RVs. Furthermore, San Segundo pinpoints two particular aspects where RV pattern variations were established, and those are primarily gender and characterization.

Put more accurately, it has been shown that Dickens frequently turned to RVs like thunder, boast, or shout in order to more successfully associate male characters with traditionally masculine traits such as strength, self-confidence, and leadership. On the other hand, these characteristics were contrasted with those of fragileness, subservience, weakness, or emotional sensitivity, which the Victorian author associated with female characters by employing verbs like sob, languish, moan, and so on.

The impact of phonically descriptive RVs is highly visible in the formation of characters such as Bill Sikes from Oliver Twist, Uriah Heep from David Copperfield, or Dodge Orlick from Great Expectations. What San Segundo (2017) implies under characterization is, for instance, the use of ASRVs as stylistic devices emphasizing the darkness of evil characters. Consequently, Dickens's villains are wont to croak, roar, or snarl, for example, which only makes their baseness more prominent to the reader. In numerous situations, the villains are thus depicted as incapable of communicating and interacting as civilized human beings, and instead reduced to the level of the animalistic, even bestial. 
Taking into account the abovementioned, several questions arise with respect to animal sounds as RVs, of which this paper attempts to raise awareness in translation studies:

1) How often is the animal feature of such verbs retained in certain translations (specifically from English into BCMS)?

2) How is its role treated in translations?

3) Is it simply glossed over with a non-animal 'noise' verb with a visceral meaning?

4) If it is disregarded, which procedures and what kind of equivalence are employed by translators in the target language (Newmark, 1988: 68-93; Baker, 2006: 10-12, 15-16; Koller, 1995: 191-222)?

\section{ANALYSIS}

The analysis was conducted on an electronic corpus which included ASRVs from Charles Dickens's novels Oliver Twist, Great Expectations, and David Copperfield. ${ }^{1}$ In the given works, the following ASRVs were used: bellow, croak, growl, roar, and shriek. Though present in far higher numbers when structures other than RVs are taken into account, they were detected in 43 instances as ASRVs. They were all checked against their BCMS translations; during this process, componential analysis was employed in order to determine the absence, that is, existence of the animal sense component of the lexical unit in the TL.

The succeeding phase involved determining if the translations which retained this component accurately reflected the sound in the SL. Because of the significance of animal sounds on characterization, it was necessary to check if the translations which retained the animal feature accurately conveyed the intended RV sound. Finally, translations not marked as precise equivalents were then analyzed in order to determine the translation procedure employed. The following table shows the verbs detected and analyzed, the novels in which they were detected, their context, their translation into BCMS, and which of those were treated as precise equivalents (marked with '+', whereas other instances were marked with '-').

\footnotetext{
${ }^{1}$ Because of the date of publication, the translations used in this analysis will be referred to as BCMS translations instead of Serbian or Serbo-Croatian.
} 
Table 3: ASRVs and their translations into BCMS

\begin{tabular}{|c|c|c|c|c|c|c|}
\hline Word & Novel & Pg. & EN & BCMS & Pg. & \\
\hline bellow & GE & 237 & $\begin{array}{l}\text { 1. He faintly moaned, 'I } \\
\text { am done for,' as the } \\
\text { victim, and he } \\
\text { barbarously bellowed, } \\
\text { 'I'll serve you out,' as } \\
\text { the murderer. }\end{array}$ & $\begin{array}{l}\text { Kao žrtva je iznemoglim } \\
\text { glasom jaukao: »Svršeno } \\
\text { je!« a kao ubojica okrutno } \\
\text { vikao: »Ubit ću te!«. }\end{array}$ & 129 & - \\
\hline croak & OT & 295 & $\begin{array}{l}\text { 2. 'Nancy, dear!' } \\
\text { croaked the Jew, in his } \\
\text { usual voice. 'Did you } \\
\text { mind me, dear?' }\end{array}$ & $\begin{array}{l}\text { Nensi, čedo moje! - } \\
\text { zamumla Jevrejin svojim } \\
\text { uobičajenim glasom. - } \\
\text { Valjda se ne ljutiš na } \\
\text { mene? }\end{array}$ & 145 & - \\
\hline \multirow{8}{*}{ growl } & DC & 164 & $\begin{array}{l}\text { 3. 'No - no,' growled } \\
\text { Mr. Barkis, reflecting } \\
\text { about it. }\end{array}$ & $\begin{array}{l}\gg \mathrm{Ne} \text {, nisam«, progunđa } \\
\text { gospodin Barkis } \\
\text { razmišljajući o tome. }\end{array}$ & 82 & - \\
\hline & DC & 207 & $\begin{array}{l}\text { 4. 'But really and truly, } \\
\text { you know. Are you?' } \\
\text { growled Mr. Barkis, } \\
\text { sliding nearer to her on } \\
\text { the seat... }\end{array}$ & $\begin{array}{l}\text { »Ama je l’ zbilja i zaista, } \\
\text { razumete li? Dobro se } \\
\text { osećate?« promumla } \\
\text { Barkis, dok joj se } \\
\text { primicao na sedištu... }\end{array}$ & 101 & - \\
\hline & DC & 208 & $\begin{array}{l}\text { 5. 'I say,' growled Mr. } \\
\text { Barkis, 'it was all right.' }\end{array}$ & $\begin{array}{l}\text { »Slušajte«, promrmlja } \\
\text { Barkis, »sve je bilo u } \\
\text { redu«. }\end{array}$ & 101 & - \\
\hline & DC & 1130 & $\begin{array}{l}\text { 6. 'Stop!' he growled to } \\
\text { me; and wiped his hot } \\
\text { face with his hand. }\end{array}$ & $\begin{array}{l}\text { »Stojte!« zareža on prema } \\
\text { meni i obrisa rukom } \\
\text { zažareno lice. }\end{array}$ & 499 & + \\
\hline & GE & 68 & $\begin{array}{l}\text { 7. No one seemed } \\
\text { surprised to see him, or } \\
\text { [...] spoke a word, } \\
\text { except that somebody in } \\
\text { the boat growled as if to } \\
\text { dogs, 'Give way, you!' } \\
\text {... }\end{array}$ & $\begin{array}{l}\text { Čini se da se nitko nije } \\
\text { iznenadio što ga vidi, niti } \\
{[\ldots] \text { je itko prozborio riječ, }} \\
\text { samo što netko povika } \\
\text { kao da se izdire na pse: - } \\
\text { Ej vi, uprite! ... }\end{array}$ & 39 & - \\
\hline & GE & 200 & $\begin{array}{l}\text { 8. 'Shall if I like,' } \\
\text { growled Orlick. }\end{array}$ & $\begin{array}{l}\text { Hoću, ako me bude volja } \\
\text { - progunđa Orlick. }\end{array}$ & 110 & - \\
\hline & GE & 201 & $\begin{array}{l}\text { 9. 'You're a foul shrew, } \\
\text { Mother Gargery,' } \\
\text { growled the } \\
\text { journeyman. }\end{array}$ & $\begin{array}{l}\text { — Vi ste pogana } \\
\text { nadžakbaba, majko } \\
\text { Gargery — zareža } \\
\text { nadničar. }\end{array}$ & 111 & + \\
\hline & GE & 202 & $\begin{array}{l}\text { 10. 'Ah-h-h!' growled } \\
\text { the journeyman, } \\
\text { between his teeth, 'I'd } \\
\text { hold you, if you was my } \\
\text { wife.' }\end{array}$ & $\begin{array}{l}\text { Ahhh! - promrsi radnik } \\
\text { kroz zube. —Ja bih vas } \\
\text { već držao da ste mi žena. }\end{array}$ & 111 & - \\
\hline
\end{tabular}




\begin{tabular}{|c|c|c|c|c|c|}
\hline GE & 210 & $\begin{array}{l}\text { 11. Orlick sometimes } \\
\text { growled, 'Beat it out, } \\
\text { beat it out - Old Clem! } \\
\text {...' }\end{array}$ & $\begin{array}{l}\text { Orlick bi ponekad } \\
\text { zarežao: 》Kujte to } \\
\text { gvožde, da iskra skače-o } \\
\text { Stari Cleme! ... }\end{array}$ & 116 & + \\
\hline GE & 233 & $\begin{array}{l}\text { 12. 'Halloa!' he } \\
\text { growled, 'where are you } \\
\text { two going?' }\end{array}$ & $\begin{array}{l}\text { Ho ho! - zareža. - Kuda } \\
\text { ćete vas dvoje? }\end{array}$ & 127 & + \\
\hline GE & 381 & $\begin{array}{l}\text { 13. 'I don't want to } \\
\text { know what passed } \\
\text { between Herbert there } \\
\text { and you,' growled } \\
\text { Drummle. }\end{array}$ & $\begin{array}{l}\text { Ne želim znati što se } \\
\text { dogodilo između Herberta } \\
\text { i vas - progunđa } \\
\text { Drummle. }\end{array}$ & 209 & - \\
\hline GE & 403 & $\begin{array}{l}\text { 14. 'And don't blame } \\
\text { me,' growled the } \\
\text { convict I had } \\
\text { recognized. }\end{array}$ & $\begin{array}{l}\text { Nemojte mene kriviti - } \\
\text { progunđa robijaš, kojeg } \\
\text { sam prepoznao. }\end{array}$ & 221 & - \\
\hline GE & 406 & $\begin{array}{l}\text { 15. 'More fool you,' } \\
\text { growled the other. }\end{array}$ & $\begin{array}{l}\text { Još si veća budala od } \\
\text { njega — progunđa drugi. }\end{array}$ & 222 & - \\
\hline GE & 451 & $\begin{array}{l}16 . . . . \text { a sulky man who } \\
\text { had been long cooling } \\
\text { his impatient nose } \\
\text { against an iron bar in the } \\
\text { front row of the gallery, } \\
\text { growled, 'Now the } \\
\text { baby's put to bed let's } \\
\text { have supper!' }\end{array}$ & $\begin{array}{l}\text {...neki je mrzovoljasti } \\
\text { gledalac u prvom redu } \\
\text { galerije, pritisnuvši i } \\
\text { hladeći nos na željeznoj } \\
\text { ogradi, nestrpljivo } \\
\text { zarežao: » Sad, kad će } \\
\text { beba na spavanje, hajdemo } \\
\text { na večeru! « }\end{array}$ & 247 & + \\
\hline OT & 24 & $\begin{array}{l}\text { 17. Mr. Gamfield } \\
\text { growled a fierce } \\
\text { imprecation on the } \\
\text { donkey generally, but } \\
\text { more particularly on his } \\
\text { eyes... }\end{array}$ & $\begin{array}{l}\text { Gospodin Gemfild } \\
\text { žestoko opsova magare } \\
\text { uopšte, a posebno njegove } \\
\text { oči... }\end{array}$ & 19 & - \\
\hline OT & 119 & $\begin{array}{l}\text { 18. 'Swear the man,' } \\
\text { growled Mr. Fang. with } \\
\text { a very ill grace. 'Now, } \\
\text { man, what have you got } \\
\text { to say?' }\end{array}$ & $\begin{array}{l}\text { Zakunite ovoga čoveka- }- \\
\text { progunđa gospodin Feng } \\
\text { veoma ljutito. - Dakle, } \\
\text { čoveče, šta imate da } \\
\text { izjavite? }\end{array}$ & 63 & - \\
\hline OT & $\begin{array}{c}137 \\
- \\
138\end{array}$ & $\begin{array}{l}\text { 19. 'Why, what the } \\
\text { blazes is in the wind } \\
\text { now!' growled a deep } \\
\text { voice. 'Who pitched that } \\
\text { 'ere at me? }\end{array}$ & $\begin{array}{l}\text { Hej, kakva je to gužva, } \\
\text { trista mu muka! - zareža } \\
\text { neki debeli glas. - Ko li } \\
\text { me je to raspalio? }\end{array}$ & 72 & + \\
\hline OT & 138 & $\begin{array}{l}\text { 20. 'Come in, d'ye } \\
\text { hear?' growled this } \\
\text { engaging ruffian. }\end{array}$ & $\begin{array}{l}\text { — Ulazi kad ti kažem! —- } \\
\text { promumla taj krasni } \\
\text { razbojnik. }\end{array}$ & 72 & - \\
\hline
\end{tabular}




\begin{tabular}{|c|c|c|c|c|c|}
\hline OT & 166 & $\begin{array}{l}\text { 21. 'Didn't know, you } \\
\text { white-livered thief!' } \\
\text { growled Sikes. } \\
\text { 'Couldn't you hear the } \\
\text { noise?' }\end{array}$ & $\begin{array}{l}\text { Nisi znao, lopove matori! } \\
\text { _ zagrme Sajks. — Zar } \\
\text { nisi čuo galamu? }\end{array}$ & 84 & - \\
\hline OT & 174 & $\begin{array}{l}\text { 22. 'Do you hear?' } \\
\text { growled Sikes, as Oliver } \\
\text { hesitated, and looked } \\
\text { round. }\end{array}$ & $\begin{array}{l}\text { Čuješ li ti šta ti kažem? - } \\
\text { promrmlja Sajks, budući } \\
\text { je Oliver oklevao i } \\
\text { obazirao se oko sebe. }\end{array}$ & 88 & - \\
\hline OT & 200 & $\begin{array}{l}\text { 23. 'You old women } \\
\text { never believe anything } \\
\text { but quack-doctors, and } \\
\text { lying story-books,' } \\
\text { growled Mr. Grimwig. }\end{array}$ & $\begin{array}{l}\text { Vi stare žene verujete } \\
\text { samo u vračare i u prazne } \\
\text { priče — progunđa } \\
\text { gospodin Grimvig. }\end{array}$ & 100 & - \\
\hline OT & 237 & $\begin{array}{l}\text { 24. 'Now, then!' } \\
\text { growled Sikes, as Oliver } \\
\text { started up; 'half past } \\
\text { five! Look sharp, or } \\
\text { you'll get no breakfast; } \\
\text { for it's late as it is.' }\end{array}$ & $\begin{array}{l}\text { Hajde, diži se! - } \\
\text { promrmlja Sajks kad se } \\
\text { Oliver trgao iza sna. - } \\
\text { Pola šest je! Požuri ili ćeš } \\
\text { ostati bez doručka; i inače } \\
\text { smo već zadocnili. }\end{array}$ & 117 & - \\
\hline OT & 315 & $\begin{array}{l}\text { 25. 'I wish I was among } \\
\text { some of you; you'd } \\
\text { howl the hoarser for it.' } \\
\text { As Sikes growled forth } \\
\text { this imprecation, with } \\
\text { the most desperate } \\
\text { ferocity that his } \\
\text { desperate nature was } \\
\text { capable of... }\end{array}$ & $\begin{array}{l}\text { — Da mi samo koji od } \\
\text { vas dopadne šaka, onda bi } \\
\text { bar znao zašto laje. } \\
\text { Psujući tako i grdeći u } \\
\text { najvećem besu za koji je } \\
\text { bila sposobna njegova } \\
\text { divlja priroda... }\end{array}$ & 155 & - \\
\hline OT & 363 & $\begin{array}{l}26 . . . . \text { twisting himself, } \\
\text { dexterously, from the } \\
\text { doctor's grasp, growled } \\
\text { forth a volley of horrid } \\
\text { oaths, and retired into } \\
\text { the house. }\end{array}$ & $\begin{array}{l}\text {... izvivši se vešto iz } \\
\text { lekarevih ruku, zasu ga } \\
\text { čitavom bujicom groznih } \\
\text { psovki i povuče se u kuću. }\end{array}$ & 176 & - \\
\hline OT & 449 & $\begin{array}{l}\text { 27. 'Oh! you've thought } \\
\text { better of it, have you?' } \\
\text { growled Sikes, marking } \\
\text { the tear which trembled } \\
\text { in her eye. }\end{array}$ & $\begin{array}{l}\text { A, sad si nesto bolje } \\
\text { smislila, je li? - gunđao } \\
\text { je Sajks opazivši suzu koja } \\
\text { joj se zavrtela u oku. }\end{array}$ & 216 & - \\
\hline OT & 453 & $\begin{array}{l}28 . \text {... but I couldn't } \\
\text { help it, upon my } \\
\text { honour.' 'Upon your } \\
\text { what?' growled Sikes, } \\
\text { with excessive disgust. }\end{array}$ & $\begin{array}{l}\text {... ali ti nisam mogao } \\
\text { priteći u pomoć, časnu ti } \\
\text { reč dajem. - Šta mi } \\
\text { daješ? - promumla } \\
\text { Sajks s izrazom krajnjeg } \\
\text { gađenja na licu. }\end{array}$ & 218 & - \\
\hline
\end{tabular}




\begin{tabular}{|c|c|c|c|c|c|c|}
\hline & OT & 483 & $\begin{array}{l}\text { 29. 'A bad one! I'll eat } \\
\text { my head if he is not a } \\
\text { bad one,' growled Mr. } \\
\text { Grimwig, speaking by } \\
\text { some ventriloquial } \\
\text { power... }\end{array}$ & $\begin{array}{l}\text { Nevaljalac je to! Poješću } \\
\text { svoju glavu ako nije } \\
\text { nevaljalac — progunđa } \\
\text { gospodin Grimving } \\
\text { govoreći nekako iz } \\
\text { trbuha... }\end{array}$ & 233 & - \\
\hline & OT & 484 & $\begin{array}{l}\text { 30. 'Do not heed my } \\
\text { friend, Miss Maylie,' } \\
\text { said Mr. Brownlow, 'he } \\
\text { does not mean what he } \\
\text { says.' 'Yes, he does,' } \\
\text { growled Mr. Grimwig. }\end{array}$ & $\begin{array}{l}\text { Ne obraćajte pažnju na } \\
\text { moga prijatelja, gospođice } \\
\text { Mejli, — reče gospodin } \\
\text { Braunlo — i on ne misli } \\
\text { kao što govori. — Jeste, } \\
\text { misli — progunđa } \\
\text { gospodin Grimvig. }\end{array}$ & 233 & - \\
\hline & OT & 484 & $\begin{array}{l}\text { 31. 'He'll eat his head, if } \\
\text { he doesn't,' growled } \\
\text { Mr. Grimwig. 'He } \\
\text { would deserve to have it } \\
\text { knocked off, if he does,' } \\
\text { said Mr. Brownlow. }\end{array}$ & $\begin{array}{l}\text { On će pojesti svoju glavu, } \\
\text { ako ne misli - gunđao je } \\
\text { gospodin Grimvig. - Ako } \\
\text { tako zaista misli, onda } \\
\text { neko treba da mu je zaista } \\
\text { razbije — reče gospodin } \\
\text { Braunlo. }\end{array}$ & 233 & - \\
\hline & OT & 527 & $\begin{array}{l}\text { 32. 'Obstinacy; } \\
\text { woman's obstinacy, I } \\
\text { suppose, my dear.' } \\
\text { 'Well, I suppose it is,' } \\
\text { growled Sikes. }\end{array}$ & $\begin{array}{l}\text { Inat, ženski inat, mislim, } \\
\text { rode moj. — To je, i ništa } \\
\text { drugo — progunđa Sajks. }\end{array}$ & 255 & - \\
\hline & OT & 568 & $\begin{array}{l}\text { 33. 'Coming!' cried the } \\
\text { office keeper, running } \\
\text { out. 'Coming,' growled } \\
\text { the guard. }\end{array}$ & $\begin{array}{l}\text { Evo idem! — uzviknu } \\
\text { upravnik poštanske stanice } \\
\text { trčeći. — Ideš — } \\
\text { progunđa sprovodnik. }\end{array}$ & 276 & - \\
\hline \multirow{3}{*}{ roar } & GE & 451 & $\begin{array}{l}34 . . . . \text { on the question } \\
\text { whether 'twas nobler in } \\
\text { the mind to suffer, some } \\
\text { roared yes, and some } \\
\text { no... }\end{array}$ & $\begin{array}{l}\text {...na pitanje je li } \\
\text { plemenitije u duši pritajiti } \\
\text { bol, jedni urlali } \gg \mathrm{Da} \ll \text {, a } \\
\text { drugi: } \gg \mathrm{Ne} \ll \text {... }\end{array}$ & 248 & - \\
\hline & OT & 107 & $\begin{array}{l}35 . . . \text { so away he went } \\
\text { like the wind, with the } \\
\text { old gentleman and the } \\
\text { two boys roaring and } \\
\text { shouting behind him. } \\
\text { 'Stop thief! Stop thief!' }\end{array}$ & $\begin{array}{l}\text {...te je jurio kao vetar sa } \\
\text { starim gospodinom i } \\
\text { dvojicom dečaka koji su } \\
\text { vikali i hajkali za njim. } \\
\text { „Drž’te lopova! Drž’te } \\
\text { lopova!“ }\end{array}$ & 57 & - \\
\hline & OT & 133 & $\begin{array}{l}\text { 36. 'Ha! ha! ha!' roared } \\
\text { Charley Bates. }\end{array}$ & $\begin{array}{l}\text { - Ha, ha, ha! - kikotao } \\
\text { se Čarli Bejts. }\end{array}$ & 69 & - \\
\hline
\end{tabular}




\begin{tabular}{|c|c|c|c|c|c|c|}
\hline & OT & 354 & $\begin{array}{l}\text { 37. He was smoking his } \\
\text { pipe here, late at night, } \\
\text { when all of a sudden } \\
\text { Chickweed roars out, } \\
\text { 'Here he is! Stop thief! } \\
\text { Murder!' }\end{array}$ & $\begin{array}{l}\text { Pušio je on tako svoju lulu } \\
\text { do duboko u noć, kad se } \\
\text { odjedanput zaorio } \\
\text { Čikvidov glas: ,Evo ga! } \\
\text { Drž’te lopova! Ubicu!' }\end{array}$ & 172 & - \\
\hline & OT & 354 & $\begin{array}{l}\text { 38. Away goes Spyers; } \\
\text { on goes Chickweed; } \\
\text { round turns the people; } \\
\text { everybody roars out, } \\
\text { 'Thieves!' and ... }\end{array}$ & $\begin{array}{l}\text { Spajers se nadade za njim, } \\
\text { a Čikvid trči li, trči; svet } \\
\text { se uskomešao, svi viču } \\
\text {,Drž’te lopova!'... }\end{array}$ & 172 & - \\
\hline & OT & 354 & $\begin{array}{l}\text { 39. At last, he couldn't } \\
\text { help shutting 'em, to } \\
\text { ease 'em a minute; and } \\
\text { the very moment he did } \\
\text { so, he hears Chickweed } \\
\text { a-roaring out, 'Here he } \\
\text { is!' }\end{array}$ & $\begin{array}{l}\text { Najzad je morao da } \\
\text { zažmuri da ih malo } \\
\text { odmori; i tek što ih je } \\
\text { zatvorio čuo je Čikvida } \\
\text { kako urla ,Drž'te ga!' }\end{array}$ & 172 & - \\
\hline & $\mathrm{OT}$ & 505 & $\begin{array}{l}\text { 40. 'Ha! ha!' roared } \\
\text { Mr. Claypole, kicking } \\
\text { up his legs in an ecstasy. }\end{array}$ & $\begin{array}{l}\text { - Ha! ha! - smejao se } \\
\text { grohotom i gospodin } \\
\text { Klejpol izbacujući noge } \\
\text { uvis od silne } \\
\text { razdraganosti. }\end{array}$ & 243 & - \\
\hline & OT & 600 & $\begin{array}{l}\text { 41. Some shouted to } \\
\text { those who were nearest } \\
\text { to set the house on fire; } \\
\text { others roared to the } \\
\text { officers to shoot him } \\
\text { dead. }\end{array}$ & $\begin{array}{l}\text { Jedni su dovikivali onima } \\
\text { koji su bili bliže da zapale } \\
\text { kuću; drugi su grmeli } \\
\text { tražeći od policajaca da } \\
\text { pucaju na ubicu. }\end{array}$ & 291 & - \\
\hline \multirow[t]{2}{*}{ shriek } & GE & 544 & $\begin{array}{l}\text { 42. 'But to be proud and } \\
\text { hard to me!' Miss } \\
\text { Havisham quite } \\
\text { shrieked, as she } \\
\text { stretched out her arms. }\end{array}$ & $\begin{array}{l}\text { Ali ponosita i okrutna } \\
\text { prema meni! — gotovo } \\
\text { cikne gospođica } \\
\text { Havisham, ispruživši obje } \\
\text { ruke. }\end{array}$ & 302 & + \\
\hline & OT & 599 & $\begin{array}{l}\text { 43. 'Help!' shrieked the } \\
\text { boy in a voice that rent } \\
\text { the air. }\end{array}$ & $\begin{array}{l}\text { — U pomoć! — vrištao je } \\
\text { dečak glasom koji je parao } \\
\text { vazduh. }\end{array}$ & 291 & - \\
\hline
\end{tabular}

\section{RESULTS AND DISCUSSION}

As can be seen in the table, bellow was used as an RV in $\mathbf{1}$ instance, croak in 1 instance, roar in 8 instances, shriek in 2, but the most striking was the use of growl as an ASRV, found no fewer than $\mathbf{3 1}$ times. Of the $\mathbf{4 3}$ given cases, upon analysis, $7 \mathbf{( 1 6 . 3 \% )}$ were noted as precise equivalents, as opposed to the remaining $36(\mathbf{8 3 . 7 \%})$, which were cases of: 
1) mistranslation - as many as $21 \mathrm{RVs}(48.9 \%)$ were mistranslated (e.g. growl as gunđati; roar as kikotati se); ${ }^{2}$

2) synonymy - detected in 11 cases $(25.6 \%)$ (e.g. roar as urlati);

3) transposition - detected in 6 cases $(13.9 \%)$ (e.g. growl forth as zasuti); ${ }^{3}$ or

4) descriptive equivalence - detected in 1 case (2.3\%) (e.g. roar as smejati se grohotom).

The given numbers depict an obvious negation of our initial hypothesis. The animal feature appears to be vulnerable in the analyzed translations into BCMS, with fewer than a fifth $(16.3 \%)$ of the ASRVs being translated with a precise equivalent and retaining this feature. The remaining $83.7 \%$ of the translations marked with "-." in the table excluded the animal sense component and they substituted ASRVs with human noise verbs (cf. Table 1).

Another noticeable detail is that mistranslations outnumber not only precise equivalents, but also the cases of descriptive equivalence, transposition, and even synonymy. Almost a half of all 43 translations disregarded the animal feature, but also failed to reflect the presence and intensity of the visceral element (e.g. growl translated as mumlati is missing elements of volume, aggressiveness, etc.) contained within the source-language RV. On the other hand, through synonymy, only slightly over a quarter of the translations show the attempt to at least preserve the visceral element if not the intended animal sound. In his elaboration on synonymy as a translation procedure, Newmark states that "a synonym is only appropriate where literal translation is not possible and because the word is not important enough for componential analysis" (1988: 84). If we view the translations of the given 43 ASRVs in the light of this statement, we reach the conclusion that resorting to synonymy was unjustified due to the fact that the given noise verbs all have more precise equivalents in BCMS (e.g. bellow and roar as rikati, croak as kreketati or graktati, growl as režati, and shriek as kreštati). In light of Newmark's elaboration, we can also point out the possibility of synonymy being applied in the TL texts precisely because of the translators' underestimation of the ASRVs' role (or at least of their animal sense component) in the SL text.

It should be mentioned that the analysis was not as clear-cut as it may seem, as was the case with the translations of the verb growl. In three situations, this RV

\footnotetext{
${ }^{2}$ Not only did they disregard the animal sense component, but they also failed to precisely include the visceral element present in the SL reporting verb.

${ }^{3}$ Of the 6 examples of transposition, 3 were part of couplets (combined with synonymy).
} 
was translated with the phonically descriptive verb (pro)mumlati, two of which were in Oliver Twist. The first association of the BCMS verb is a human sound, although, upon checking its dictionary definition, it was discovered that the word mumlati can also refer to a sound produced by bears, for example. ${ }^{4}$

Consequently, a dilemma arose as to whether the translation of growl as mumlati was to be treated in the analysis as one retaining the animal sense component in these particular works. One of the ways to avoid the danger of subjectivity in that analysis was to look at all the instances of the verb mumlati as a translation of any occurring ASRVs and check for any existing patterns in its employment. Thus, the dilemma was resolved upon looking into the solution of the verb croak, which was also translated with the verb mumlati. This was a strong indicator that animal sounds such as growl and croak were not differentiated in translations and that, in the case of the verb mumlati, the focus was not kept on (the nuancing of) the animal sense component. Instead, a human sound was consistently used and is present in most cases throughout the translation (we also notice the synonyms (pro)gunđati, (pro)mrmljati, and promrsiti as translations of growl).

The aforementioned dilemma also led to the possible treatment of the verb (pro)mumlati as a translation of growl as an instance of synonymy, in which case the verbs (pro)mrmljati and promrsiti could have been seen as more acceptable solutions, as they are synonymous with the verb (pro)mumlati. Nevertheless, as previously elaborated, the misrepresentation of the animal feature was determined, resulting in the RV (pro)mumlati being treated as a case of mistranslation. Furthermore, if back-translated, (pro)mumlati, (pro)gunđati, (pro)mrmljati, and promrsiti end up in English as mumble, grumble, and mutter. Though some of these verbs do convey a note of displeasure or negativity, they do not remain faithful to the far more intense visceral elements of hostility and aggression, contained within the verb growl or its precise BCMS equivalent režati. Furthermore, the lack of the animal feature in the verbs (pro)gunđati, (pro)mrmljati, and promrsiti impacts the formation and interpretation of their respective literary characters, softening them and altering their emotional coloring. This is one of the main reasons why they are marked as mistranslations of the RV growl.

On the basis of what has been detected in these three novels, the animal sense component is frequently assigned less significance, and the same seems to

\footnotetext{
${ }^{4}$ Vujanić, M., Gortan-Premk, D., Dešić, M., et al. (2011). Rečnik srpskoga jezika. Novi Sad: Matica srpska, pp. 729, 1052.
} 
apply to the source-language RVs' visceral sense components. This is of questionable acceptability because the source-language RVs and the sounds they convey were purposefully employed by Dickens as a stylistic device with a specific function (San Segundo, 2017).

\section{CONCLUDING REMARKS AND POSSIBLE FURTHER RESEARCH}

The findings of this analysis suggest that, in the abovementioned novels, the translations of ASRVs interfere with the author's stylistic devices and their effectiveness. Considering the given suggestion, an entire topic for further research has emerged. By combining the principles of characterization in literature with an analysis of ASRVs as components of it, and by analyzing translations of English texts into BCMS, much can be learned about the skill and art of translation. First, since ASRVs lend a hue to a character's personation (i.e. how it is that he or she communicates with others), if the translation strategies employed preserve that hue, the emotional coloring will also surface in the TL. Conversely, elements of the character's personation will be lost. It follows then than further research could be done to discover how characters are perceived in the TL culture, if the readership's reaction to them is similar to that of the SL culture. Second, although the findings of this paper are interesting, it must be said that the sample used was limited. It is difficult to draw larger conclusions based on three books by one author and four translators. Using a large corpus of literary works in English that have been translated into BCMS should shed more light on the accuracy and consistency of translators.

Likewise, it should be noted that the SL texts were all at least 150 years old (the translations more than 40), and it would be useful to examine newer texts to see if the situation has changed. The lexical field of RVs is quite large in English. It would be worthwhile to examine a larger corpus to establish how large that field is in BCMS and to see whether those RVs are used with the same flexibility and frequency in BCMS texts. Moreover, it would be interesting to examine how translators of those texts into English cope with RVs of all sorts. Finally, the conclusions drawn from such studies would be quite useful to future translators, helping them to ensure accuracy and consistency. 
Ema Pandrc, Randall A. Major

\section{ŽIVOTINJSKI ZVUCI KAO UVODNI GLAGOLI U PREVODIMA SA ENGLESKOG NA SRPSKI}

Rezime

Ovaj rad se bavi upotrebom uvodnih glagola koji se odnose na životinjske zvuke kao što su režati, lajati, kreketati, graktati, itd. Takvi glagoli se koriste u književnim tekstovima radi karakterizacije likova, tj. upotrebljavaju se da bi pisac efektnije istakao određene osobine datog lika. Stoga se može reći da je njihova uloga od izrazitog značaja i da se ona treba imati u vidu prilikom prevođenja književnih dela. Značaj ovog rada se, dakle, ogleda u korisnosti uvida $\mathrm{u}$ to koliko se $\mathrm{u}$ prevodima sa engleskog vodi računa o tačnosti $\mathrm{i}$ usaglašenosti uvodnih glagola ovoga tipa.

Krenuvši od hipoteze da se u većini slučajeva prevodioci opredeljuju za precizne ekvivalente da bi sačuvali karakterizaciju likova u ciljnom tekstu, sastavili smo elektronski korpus od tri romana Čarlsa Dikensa (Oliver Tvist, Velika očekivanja i David Koperfild), kako u originalu tako i u prevodu, da bismo stekli uvid u prevodilačka rešenja. Zatim smo sastavili spisak uvodnih glagola koji sadrže životinjske zvuke na engleskom i proverili koji od njih se javljaju u gorepomenutim romanima $u$ originalu. Naredni korak je zahtevao obeležavanje i izdvajanje svih rečenica u kojima su pronađeni životinjski uvodni glagoli, što je obezbedilo uslove za narednu fazu rada: traženje prevoda datih rečenica i izdvajanje istih zarad detaljnije analize i poređenja sa njihovim originalom.

Sledeći korak podrazumevao je vršenje analize značenjskih komponenata prevedenih uvodnih glagola, i to u cilju dolaženja do tačnog broja prevodilačkih rešenja čije je značenje zadržalo životinjsku komponentu. Naravno, da bi se rešenje smatralo preciznim ekvivalentom, bilo je potrebno da, pored zadržavanja životinjske komponente, precizno prenese u ciljni jezik zvuk koji je autor zamislio. Poslednja faza analize predstavljala je određivanje prevodilačkih procedura koje su prevodioci upotrebili u slučajevima koje nismo tretirali kao precizne ekvivalente.

Engleski glagoli koje smo pronašli u ovim romanima su bellow, croak, growl, roar, i shriek, sve skupa upotrebljenih kao uvodni glagoli u 43 slučaja. Od toga, samo 16,3\% činili su precizni ekvivalenti, dok je životinjska značenjska komponenta bila izuzeta u 83,7\% slučajeva, što se primetno kosi sa uspostavljenom hipotezom. Naročito je interesantan i još jedan podatak izveden iz analize, a to je da je gotovo polovina glagola $(48,9 \%)$ pogrešno prevedena. Zatim sledi upotreba sinonimije kao prevodilačke procedure, koja je prisutna u $25,6 \%$ slučajeva, transpozicija (13,9\%, od kojih je pola kombinovano sa sinonimijom) i deskriptivna ekvivalencija (2,3\%, tj. samo jedan slučaj). Nizak procenat slučajeva sinonimije i visok procenat pogrešnih prevoda takođe pokazuju da, pored životinjske komponente, zanemareno je i ophođenje i sveukupno psihofizičko stanje lika. Date brojke nas stoga dovode do zaključka da je uloga ove vrste uvodnih glagola potcenjena $u$ 
prevodima i da se odsustvo određenih značenjskih komponenti negativno odražava na njihov učinak kao stilskog sredstva. Posledica tog negativnog uticaja je ublažavanje i ometanje karakterizacije književnih likova.

Ključne reči: uvodni glagoli, životinjski zvukovi, karakterizacija, književna dela, prevodilačke procedure, značenjske komponente

\section{SOURCES}

Dickens, C. (1850). David Copperfield. London: Bradbury and Evans.

Dickens, C. (1861). Great Expectations. London: Chapman and Hall.

Dickens, C. (1838). Oliver Twist. London: Richard Bentley.

Dikens, Č. (1974). David Koperfild. Beograd: Nolit. (translated by Mihailo Đorđević \& Jugoslav Đorđević).

Dikens, Č. (2005). Velika Očekivanja. Zagreb: Globus media. (translated by Zlatko Gorjan).

Dikens, Č. (1969). Oliver Tvist. Beograd: Nolit. (translated by Božidar Marković).

Dickens, C. David Copperfield. https://www.planetebook.com/free-ebooks/davidcopperfield.pdf

Dickens, C. Great Expectations. http://www.planetpublish.com/wp-content/ uploads/2011/11/Great_Expectations_NT.pdf

Dickens, C. Oliver Twist. https://www.planetebook.com/free-ebooks/olivertwist.pdf

Dikens, ¿̌. David Koperfild. https://kupdf.net/download/268-arls-dikens-davidkoperfild_5afe6a30e2b6f50716237c27_pdf

Dikens, Č. Velika Očekivanja. https://issuu.com/vesnasavic6/docs/velika_ ocekivanja_-_charles_dickens

Dikens, Č. Oliver Tvist. https://www.scribd.com/document/332216786/\%C4\% 8Carls-Dikens-Oliver-Tvist-pdf

\section{REFERENCES}

Baker, M. (1992; 2006) In Other Words. New York, Routledge.

Brown, G. (1990). Listening to Spoken English. Harlow, U.K.: Longman Group Ltd.

Brüngel-Dittrich, M. (2005). Speech Presentation in the British and German Press. Berlin: Peter Lang. [As cited in: San Segundo, P. (2017). Reporting verbs as a stylistic device in the creation of fictional personalities in literary texts. Atlantis, 39 (2), 105-124.] 
Caldas-Coulthard, C. (1987). Reported speech in written narrative texts. In: Malcolm Coulthard (ed.) (1987). Discussing Discourse. Birmingham: University of Birmingham.149-167.

Hatim, B. and Munday, J. (2004) Translation: An Advanced Resource Book. New York: Routledge.

Koller, W. (1995). [As cited in Hatim and Munday (2004): "The Concept of Equivalence and the Object of Translation Studies", Target 7:2: 191-222.]

Leech, G. (1983). Principles of Pragmatics. London \& New York: Longman.

Newmark, P. (1988). A Textbook of Translation. Hempstead, U.K.: Prentice Hall International.

San Segundo, P. (2017). Reporting verbs as a stylistic device in the creation of fictional personalities in literary texts. Atlantis, 39 (2), 105-124.

San Segundo, P. (2018). CLiC and Dickens's not-so-conspicuous techniques of characterisation: Reporting verbs. [Blog post] Retrieved from https://blog.bham.ac.uk/clic-dickens/2018/01/13/clic-and-dickenssreporting-verbs/

Silk, D. (2007). Bee Parables. Maitland, FL: Xulon Press.

Vujanić, M.-Gortan-Premk, D.-Dešić, M., et al. (2011). Rečnik srpskoga jezika. Novi Sad: Matica srpska. 